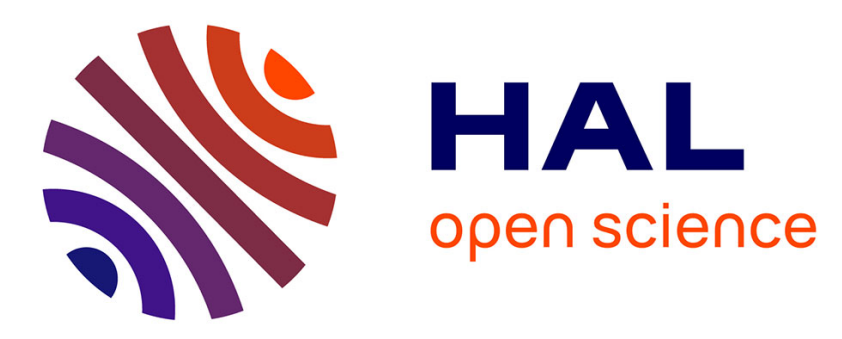

\title{
Dimensional Reduction of Cardiac Models for Effective Validation and Calibration
}

Matthieu Caruel, Radomir Chabiniok, Philippe Moireau, Yves Lecarpentier, Dominique Chapelle

\section{- To cite this version:}

Matthieu Caruel, Radomir Chabiniok, Philippe Moireau, Yves Lecarpentier, Dominique Chapelle. Dimensional Reduction of Cardiac Models for Effective Validation and Calibration. 7th International Conference on Functional Imaging and Modeling of the Heart, FIMH 2013, Jun 2013, London, United Kingdom. pp.259-267. hal-00834704

\section{HAL Id: hal-00834704 https://hal.science/hal-00834704}

Submitted on 17 Jun 2013

HAL is a multi-disciplinary open access archive for the deposit and dissemination of scientific research documents, whether they are published or not. The documents may come from teaching and research institutions in France or abroad, or from public or private research centers.
L'archive ouverte pluridisciplinaire HAL, est destinée au dépôt et à la diffusion de documents scientifiques de niveau recherche, publiés ou non, émanant des établissements d'enseignement et de recherche français ou étrangers, des laboratoires publics ou privés. 


\title{
Dimensional reduction of cardiac models for effective validation and calibration
}

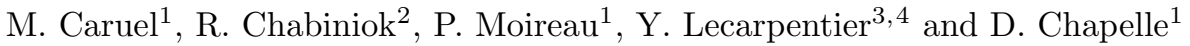 \\ 1 Inria Saclay Ile-de-France, M $\exists D I S I M$ team, Palaiseau, France \\ 2 Division of Imaging Sciences \& Biomedical Engineering, St Thomas' Hospital, \\ King's College London, UK \\ 3 Institut du Coeur, Hôpital de la Pitié-Salpêtrière, Paris, France \\ 4 Centre de Recherche Clinique, Hôpital de Meaux, France
}

\begin{abstract}
Complex 3D beating heart models are now available, but their complexity makes calibration and validation very difficult tasks. We thus propose a systematic approach of deriving simplified reduceddimensional models, in "0D" - typically, to represent a cardiac cavity, or several coupled cavities - and in "1D" - to model elongated structures such as fibers or myocytes. As illustrations of our approach, we demonstrate model validation based on experiments performed with papillary muscles, and calibration using patient-specific pressure-volume loops.
\end{abstract}

Complex 3D multi-physics beating heart models are now available [5], including for patient-specific simulations based on inverse modeling approaches [9]. However, such models are computationally intensive, and their physical and computational complexities make their detailed validations and calibrations difficult. Preliminary calibrations of the numerous physical parameters are essential to run meaningful simulations, and to initiate inverse modeling loops for personalization purposes, and it is very ineffective to perform this preliminary stage with the full 3D model. Furthermore, when subtle modeling refinements are investigated, the complete organ is not the adequate scale to assess and validate such fine effects, whereas detailed experiments are frequently available at a more local scale, namely, tissue sample [7] or even myocyte [1]. This motivates our proposed approach of systematic derivation of simplified reduced-dimensional models, both in "0D" - typically, to represent a cardiac cavity, or several coupled cavities - and in "1D" - to model elongated structures such as fibers or myocytes. Such hierarchical models are intended for use in combination with 3D models to provide dramatic effectiveness gains without compromising modeling accuracy.

\section{The 3D model}

We consider the cardiac model proposed in $[8,3]$, of which we now summarize the main ingredients, also focusing on distinctions that we introduce in this work. 
Sarcomere behavior We first concentrate on the behavior of the sarcomeres, namely, the contractile units inside myofibers. Let us denote by $e_{c}$ the sarcomere strain. All along the thick myosin filaments within the sarcomeres, myosin heads can attach to special sites located on thin actin filaments, thus creating socalled cross-bridges. For a given myosin head, we denote by $s$ the distance to the closest actin site scaled by a characteristic interspace distance. Following Huxley's description in [6], we introduce $n(s, t)$ the fraction of heads attached at a distance $s$ at time $t$. As long as the head remains attached, its extension $s$ varies at the same rate as $e_{c}$, hence,

$$
\frac{\partial n}{\partial t}+\dot{e}_{c} \frac{\partial n}{\partial s}=\left(n_{0}\left(e_{c}\right)-n\right) f-n g,
$$

where $f$ and $g$ denote binding and unbinding rates, respectively, and the strain dependent function $n_{0}$ accounts for the length-dependent fraction of recruitable myosin heads (Frank-Starling effect) [3]. We model $f$ and $g$ by

$$
f(s, t)=|u|_{+} \mathbb{1}_{s \in[0,1]}, \quad g(s, t)=|u|-f(s, t)=|u|_{+} \mathbb{1}_{s \notin[0,1]}+|u|_{-},
$$

where $u$ denotes a variable reaction rate summarizing chemical activation, inducing contraction or relaxation depending on whether $u$ is positive or negative, respectively. Relaxation being known to be load-dependent in mammalian cardiac muscles [7], we here represent this effect by a new internal variable $w(t)$ obeying the first-order dynamics

$$
\alpha_{r} \dot{w}=m_{0}\left(e_{c}\right)-w,
$$

which has a multiplicative effect when $u \leq 0$ (relaxation), namely,

$$
u=|\bar{u}(t)|_{+}-w|\bar{u}(t)|_{-},
$$

where $\bar{u}(t)$ is an input variable independent of the sarcomere state. The stress state in the sarcomere is then obtained by assuming simple quadratic energy for realized cross-bridges, in the form $W_{m}(s)=\frac{k_{0}}{2}\left(s+s_{0}\right)^{2}$, namely, a linear spring of stiffness $k_{0}$ and pre-strain $s_{0}$. The overall stiffness and stress in the sarcomere are thus given by

$$
k_{c}(t)=k_{0} \int n(s, t) d s, \quad \tau_{c}(t)=\int W_{m}^{\prime}(s) n(s, t) d s=k_{0} \int\left(s+s_{0}\right) n(s, t) d s,
$$

where we recognize the first two moments of the density function $n$. When integrating in (1), this leads to the following closed-form dynamical system

$$
\left\{\begin{array}{l}
\dot{k}_{c}=-\left(|\bar{u}|_{+}+w|\bar{u}|_{-}\right) k_{c}+n_{0} k_{0}|\bar{u}|_{+} \\
\dot{\tau}_{c}=-\left(|\bar{u}|_{+}+w|\bar{u}|_{-}\right) \tau_{c}+n_{0} \sigma_{0}|\bar{u}|_{+}+k_{c} \dot{e}_{c}
\end{array}\right.
$$

where $\sigma_{0}=k_{0}\left(s_{0}+1 / 2\right)$ represents the maximum active stress. 
Overall constitutive law In order to take into account the passive behavior, we use the Hill-Maxwell rheological law [8] and combine the above contractile behavior with a $1 \mathrm{D}$ series linearly-elastic element of stiffness $E_{s}$ along the fiber, and a 3D viscoelastic element in parallel. This provides the additional dynamical relation

$$
\tau_{c}+\mu \dot{e}_{c}=E_{s} \frac{\left(e_{1 \mathrm{D}}-e_{c}\right)\left(1+2 e_{1 \mathrm{D}}\right)}{\left(1+2 e_{c}\right)^{3}},
$$

with $\mu$ a viscous damping parameter, and $e_{1 \mathrm{D}}$ the strain component along the fiber, i.e. $e_{1 \mathrm{D}}=\underline{\tau}_{1} \cdot \underline{\underline{e}} \cdot \underline{\tau}_{1}$ where $\underline{\underline{e}}$ denotes the Green-Lagrange strain tensor. The parallel association then yields for the second Piola-Kirchhoff stress tensor

$$
\underline{\underline{\underline{\Sigma}}}=\underline{\underline{\Sigma}}_{p}+\sigma_{1 \mathrm{D}} \underline{\tau}_{1} \otimes \underline{\tau}_{1}-p \underline{\underline{C}}^{-1},
$$

with $\underline{\underline{C}}$ the Cauchy-Green strain tensor, $p$ a pressure-type Lagrange multiplier accounting for material incompressibility, and

$$
\sigma_{1 \mathrm{D}}=\frac{1+2 e_{c}}{1+2 e_{1 \mathrm{D}}}\left(\tau_{c}+\mu \dot{e}_{c}\right), \quad \underline{\underline{\Sigma_{p}}}=\frac{\partial W_{e}}{\partial \underline{\underline{e}}}(\underline{\underline{e}})+\frac{\partial W_{v}}{\partial \underline{\underline{\dot{e}}}}(\underline{\underline{e}}, \underline{\underline{\underline{e}}}),
$$

where $W_{e}$ and $W_{v}$ denote a hyperelastic potential and a viscous pseudo-potential, respectively. In the sequel, we will consider the specific form $W_{v}=\frac{\eta}{2} \operatorname{tr}(\underline{\underline{e}})^{2}$, and further elaborate on $W_{e}$.

System equilibrium The overall mechanical behavior is governed by the so-called principle of virtual work in Lagrangian formulation

$$
\int_{\Omega_{0}} \rho \underline{\ddot{y}} \cdot \underline{y}^{*} d \Omega+\int_{\Omega_{0}} \underline{\underline{\Sigma}}: d_{\underline{y}} \underline{\underline{e}} \cdot \underline{y}^{*} d \Omega=-\int_{S_{\text {endo }}} P_{v} \underline{n} \cdot \underline{\underline{F}}^{-1} \cdot \underline{y}^{*} d S, \quad \forall \underline{y}^{*} \in \mathcal{V},
$$

where $y$ denotes the displacements - with respect to a reference configuration $\Omega_{0}$ - of deformation gradient $\underline{\underline{F}}, y^{*}$ the corresponding test functions, $P_{v}$ the blood pressure exerting forces on the endocardium surfaces $S_{\text {endo }}$ of outward unit normal vector $\underline{n}$. The internal pressure $P_{v}$ is coupled to the cardiac outflow $Q=-\dot{V}$ where $V$ is the ventricular cavity volume. Inflows and outflows occur depending on the balance of internal and external pressures summarized as [8]

$$
-\dot{V}=Q=f\left(P_{v}, P_{a r}, P_{a t}\right),
$$

where $P_{a r}$ and $P_{a t}$ denote the arterial and atrial pressures, respectively. Finally, the system is closed by a relation representing the external circulation, with a so-called two-stage Windkessel model written as

$$
\left\{\begin{array}{l}
C_{p} \dot{P}_{a r}+\left(P_{a r}-P_{d}\right) / R_{p}=Q \\
C_{d} \dot{P}_{d}+\left(P_{d}-P_{a r}\right) / R_{p}=\left(P_{v s}-P_{d}\right) / R_{d}
\end{array}\right.
$$

where $C_{p}, R_{p}, C_{d}$ and $R_{d}$ denote capacitances and resistances of the proximal and distal circulations, and $P_{v s}$ is a constant representing the venous system pressure. 


\section{Reduced formulations}

\subsection{D-formulation}

(a)

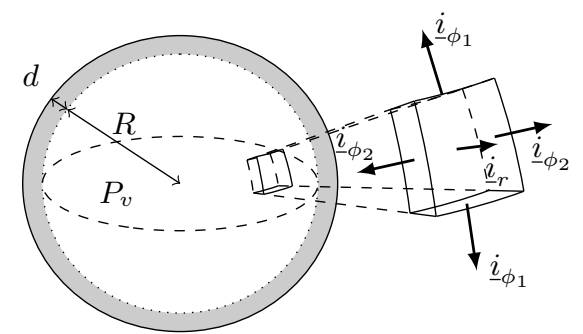

(b)

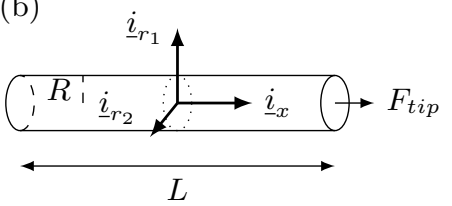

Fig. 1. Reduced models geometries. (a): 0D spherical model of a ventricle. (b): $1 \mathrm{D}$ cylindrical model of a single muscle fiber

Geometry and kinematics We define a simplified problem in which the geometry and the physical behavior are endowed with spherical symmetry properties. The simplified geometry for a ventricle is given by a sphere of radius $R_{0}$ and thickness $d_{0}$ in the stress-free reference configuration, see Fig.1 (a). Assuming that the constitutive properties also exhibit spherical symmetry, the resulting behavior under an internal pressure loading will display the same symmetry, and the deformed configuration is then characterized by dilated radius $R$ and shrunk thickness $d$. At any given location, we denote by $\left(\underline{i}_{r}, \underline{i}_{\phi_{1}}, \underline{i}_{\phi_{2}}\right)$ an orthonormal basis, with $\underline{i}_{r}$ radial and $\left(\underline{i}_{\phi_{1}}, \underline{i}_{\phi_{2}}\right)$ orthoradial defined so that $\underline{i}_{\phi_{1}}=\underline{\tau}_{1}$. With the above assumptions the displacement field with respect to the reference configuration is radial, namely, given by $y=y \underline{i}_{r}=\left(R-R_{0}\right) \underline{i}_{r}$, and the Cauchy-Green strain tensor has the special form

$$
\underline{\underline{C}}=\left(\begin{array}{ccc}
C_{r r} & 0 & 0 \\
0 & C & 0 \\
0 & 0 & C
\end{array}\right),
$$

where $C=\left(1+y / R_{0}\right)^{2}$ is the dilatation in the orthoradial plane. Assuming incompressible behavior, we have $\operatorname{det} \underline{\underline{C}}=1$, hence $C_{r r}=C^{-2}$. Considering a virtual displacement $y^{*}=y^{*} \underline{i}_{r}$ with the above-assumed kinematic symmetry, we find in each orthoradial direction $\left(d_{\underline{y}} \underline{\underline{e}} \cdot \underline{y}^{*}\right)_{\phi \phi}=\left(1+y / R_{0}\right)\left(y^{*} / R_{0}\right)$.

Stress and equilibrium derivation Due to the relative thin-ness of the wall compared to the sphere radius, classical arguments of shell theory justify that the radial stress $\Sigma_{r r}$ can be neglected compared to the orthoradial components [2]. Therefore, in the decomposition (5) the Lagrange multiplier $p$ can be explicitly inferred from $\Sigma_{r r}=0$, viz.

$$
p=C^{-2}\left(\underline{\underline{\Sigma}}_{p}\right)_{r r} .
$$

Hence, in (7) the power of internal forces per unit volume gives

$$
\underline{\underline{\Sigma}}: d_{\underline{y}} \underline{\underline{e}} \cdot \underline{y}^{*}=\left(1+\frac{y}{R_{0}}\right) \frac{y^{*}}{R_{0}} \Sigma_{s p h},
$$


with the combined stress quantity $\Sigma_{s p h}$ defined by

$$
\Sigma_{s p h}=\left(\underline{\underline{\Sigma}}_{p}\right)_{\phi_{1} \phi_{1}}+\left(\underline{\underline{\Sigma}}_{p}\right)_{\phi_{2} \phi_{2}}+\sigma_{1 \mathrm{D}}-2 C^{-3}\left(\underline{\underline{\Sigma}}_{p}\right)_{r r} .
$$

The passive stress $\underline{\underline{\Sigma}}_{p}$ depends on the specific choice of the energy functionals $W_{e}$ and $W_{v}$, see (6). Here, we consider a hyperelastic potential of transverse isotropic type $W_{e}\left(J_{1}, J_{2}, J_{4}\right)$, function of the classical reduced invariants of the Cauchy-Green tensor given here by

$$
\left\{\begin{array}{l}
J_{1}=\operatorname{tr}(\underline{\underline{C}})(\operatorname{det} \underline{\underline{C}})^{-1 / 3}=2 C+C^{-2} \\
J_{2}=\frac{1}{2}\left(\operatorname{tr}(\underline{\underline{C}})^{2}-\operatorname{tr}(\underline{\underline{C}})\right)(\operatorname{det} \underline{\underline{C}})^{-2 / 3}=C^{2}+2 C^{-1} \\
J_{4}=\underline{\tau}_{1} \cdot \underline{\underline{C}} \cdot \underline{\tau}_{1}(\operatorname{det} \underline{\underline{C}})^{-1 / 3}=\bar{C}
\end{array}\right.
$$

In order to derive the passive stress $\underline{\underline{\Sigma}}_{p}$, for the hyperelastic part we use the chain rule $\partial W_{e} / \partial \underline{\underline{e}}=2 \sum_{i}\left(\partial W_{e} / \partial J_{i}\right)\left(\partial J_{i} / \partial \underline{\underline{C}}\right)$, where classical expressions of the invariant derivatives yield

$$
\left\{\begin{array}{l}
\frac{\partial J_{1}}{\partial \underline{\underline{C}}}=\underline{\underline{I}}-\frac{1}{3}\left(2 C+C^{-2}\right) \underline{\underline{C}}^{-1} \\
\frac{\partial \overline{J_{2}}}{\partial \underline{\underline{C}}}=\left(2 C+C^{-2}\right) \underline{\underline{I}}-\underline{\underline{C}}-\frac{2}{3}\left(C^{2}+2 C^{-1}\right) \underline{\underline{C}}^{-1} \\
\frac{\partial \overline{J_{4}}}{\partial \underline{\underline{C}}}=\underline{i}_{\phi_{1}} \otimes \underline{i}_{\phi_{1}}-\frac{1}{3} C \underline{\underline{C}}^{-1}
\end{array}\right.
$$

while the viscous contribution gives $\partial W_{v} / \partial(\underline{\underline{e}})=\eta \underline{\underline{\dot{e}}}$. Substituting into (13) we obtain after simplifications

$$
\Sigma_{s p h}=\sigma_{1 \mathrm{D}}+4\left(1-C^{-3}\right)\left(\frac{\partial W_{e}}{\partial J_{1}}+C \frac{\partial W_{e}}{\partial J_{2}}\right)+2 \frac{\partial W_{e}}{\partial J_{4}}+\eta \dot{C}\left(1+2 C^{-6}\right),
$$

where $\sigma_{1 \mathrm{D}}$ is given by (6). The other terms in (7) can be integrated directly, hence, we obtain the following ODE for the displacement $y$

$$
\rho d_{0} \ddot{y}+\frac{d_{0}}{R_{0}}\left(1+\frac{y}{R_{0}}\right) \Sigma_{s p h}=P_{v}\left(1+\frac{y}{R_{0}}\right)^{2} .
$$

Finally, the equation (8) can be expressed as

$$
-\dot{V}=4 \pi R_{0}^{2}\left(1+\frac{y}{R_{0}}\right)^{2} \dot{y}=f\left(P_{v}, P_{a r}, P_{a t}\right),
$$

and all the other equations of the initial 3D problem remain unchanged. Note that our reduction approach is very general and could be applied to most other 3D cardiac models for similar validation and calibration purposes, indeed. 


\subsection{D-formulation}

Geometry and kinematics This one-dimensional model aims at reproducing the behavior of an elongated structure made of myocardium, such as isolated muscle fibers, or even single myocytes, under uniaxial traction. As a simplified geometry we consider a circular cylinder of radius $R_{0}$ and length $L_{0}$ in the reference configuration, see Fig.1(b), and we assume that material properties accordingly enjoy cylindrical symmetry, hence the whole behavior has this same symmetry. As an orthonormal basis we use a first vector $\underline{i}_{x}$ oriented along the fiber - i.e. $\underline{\tau}_{1}=\underline{i}_{x}$ - and we define two arbitrary equivalent directions $\left(\underline{i}_{r_{1}}, \underline{i}_{r_{2}}\right)$ in the cross section. An external force $F_{t i p}$ is applied at the end of the fiber along the $\underline{i}_{x}$-direction, and we seek the resulting longitudinal displacement $y(x)$ at each point of the fiber. Due to the incompressibility condition, the Cauchy-Green tensor takes the special form

$$
\underline{\underline{C}}=\left(\begin{array}{ccc}
C & 0 & 0 \\
0 & C^{-\frac{1}{2}} & 0 \\
0 & 0 & C^{-\frac{1}{2}}
\end{array}\right),
$$

where $C=\left(1+y^{\prime}(x)\right)^{2}$ is the strain in the $\underline{i}_{x}$-direction.

Stress and equilibrium derivation Considering again the small thickness (diameter) of the fiber and the loading in the axial direction, classical structural mechanics justifies that the radial stresses $\Sigma_{r r}$ are negligible. Like in the 0D model reduction, this allows to compute the Lagrange multiplier $p$, viz.

$$
p=C^{-1 / 2}\left(\underline{\underline{\Sigma}}_{p}\right)_{r r} .
$$

The power of internal forces reduces to $\underline{\underline{\Sigma}}: d_{\underline{y}} \underline{\underline{e}} \cdot \underline{y}^{*}=\Sigma_{x x}\left(1+y^{\prime}\right)\left(y^{*}\right)^{\prime}$, for a virtual displacement field $\underline{y}^{*}(x)=y^{*}(x) \underline{i}_{x}$, with the axial stress given by

$$
\Sigma_{x x}=\left(\underline{\underline{\Sigma}}_{p}\right)_{x x}+E_{s} \frac{e-e_{c}}{\left(1+2 e_{c}\right)^{2}}-C^{-3 / 2}\left(\underline{\underline{\Sigma}}_{p}\right)_{r r},
$$

with $e=e_{1 D}=(C-1) / 2$. In this case, we have for the hyperelastic stress

$$
\begin{cases}J_{1}=C+2 C^{-1 / 2}, & \frac{\partial J_{1}}{\partial \underline{C}}=1-\frac{1}{3}\left(C+2 C^{-1 / 2}\right) \underline{\underline{C}}^{-1} \\ J_{2}=2 C^{1 / 2}+C^{-1}, & \frac{\partial \overline{J_{2}}}{\partial \underline{\bar{C}}}=\left(C+2 C^{-1 / 2}\right) \underline{\underline{I}}-\underline{\underline{C}}-\frac{2}{3}\left(2 C^{1 / 2}+C^{-1}\right) \underline{\underline{C}}^{-1} \\ J_{4}=C, & \frac{\partial \underline{J}_{4}}{\partial \underline{\underline{C}}}=\underline{i}_{x} \otimes \underline{i}_{x}-\frac{1}{3} C \underline{\underline{C}}^{-1}\end{cases}
$$

and the derivative of the viscous pseudo-potential gives $\frac{\partial W_{v}}{\partial \underline{\underline{\underline{e}}}}=\eta \underline{\underline{\dot{e}}}$. Then we find

$$
\Sigma_{x x}=\sigma_{1 \mathrm{D}}+2\left(1-C^{-3 / 2}\right)\left(\frac{\partial W_{e}}{\partial J_{1}}+C^{-1 / 2} \frac{\partial W_{e}}{\partial J_{2}}\right)+2 \frac{\partial W_{e}}{\partial J_{4}}+\frac{\eta}{2} \dot{C}\left(1+\frac{1}{2} C^{-\frac{9}{4}}\right) .
$$

Finally, in (7) we can integrate over each cross-section, which yields

$$
\int_{0}^{L_{0}}\left[\rho \ddot{y} y^{*}+\Sigma_{x x}\left(1+y^{\prime}\right)\left(y^{*}\right)^{\prime}\right] d x=\frac{F_{t i p}}{\pi R_{0}^{2}} y^{*}(L) .
$$




\section{Results}

Fig.2 summarizes the experimental protocol considered - designed to study the response of an isolated papillary muscle sample under loading conditions similar to a cardiac cycle, see [7] for the details of the protocol used here. We will demonstrate how the corresponding data can be employed for modeling validation and calibration, in combination with our dimensional reduction strategy.

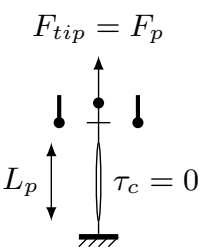

(a) passive

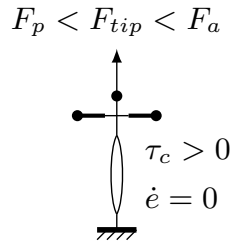

(b) isometric

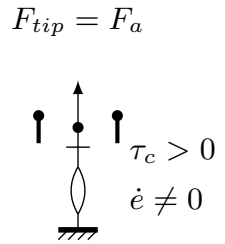

(c) isotonic

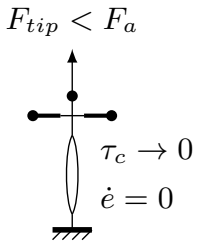

(d) isometric

Fig. 2. Experimental protocol for rat papillary muscle. (a): the fiber is stretched passively to a length $L_{p}$ by a preload $F_{p} ;($ b): electric stimulation induces a twitch contraction against an afterload $F_{a}$, further displacement being prevented by a clamp system until the force $F_{t i p}$ measured by the loading device reaches the prescribed afterload; (c): the clamp is removed to allow isotonic shortening; (d): during relaxation, the fiber elongates until it recovers the initial length $L_{p}$ where the clamp is put back.

Calibration Two data sets corresponding to two different preloads are available. Passive equilibrium under each preload is used to calibrate the parameters of the hyperelastic potential chosen in the form [4]

$$
W_{e}(\underline{\underline{e}})=C_{0} \exp \left(C_{1}\left(J_{1}(\underline{\underline{e}})-3\right)^{2}\right)+C_{2} \exp \left(C_{3}\left(J_{4}(\underline{\underline{e}})-1\right)^{2}\right) .
$$

The function $n_{0}\left(e_{c}\right)$ is calibrated from the static force balance at maximum shortening for the different afterloads. The activation $u$ is adjusted to fit the stress kinetics in the isometric case for the higher preload, see blue curves in Fig.3, (a) and (c). Finally, the relaxation parameters $\alpha_{r}$ and $m_{0}\left(e_{c}\right)$ are adjusted so that the relaxation paths correspond to experimental data.

Simulations Fig.3 (a)-(d) shows the dynamics of strain $e$ and tension $F_{t i p} / A_{0}$, as simulated by the 1D-model and compared to experimental data for the two different preloads, while Fig.3 (e) shows the resulting Hill-type force-velocity curves. Note that at time $t=0$ the system starts with an initial stretch associated with the preload considered. In Fig.4, we plot the results obtained with the 0Dmodel when using the same physical parameters as in the 1D-simulations (except for $\sigma_{0}$ ), and geometric dimensions representative of a left ventricle. In turn, the 0D simulations can be used as a pre-calibration step for a 3D model, as will be further demonstrated in future studies. 


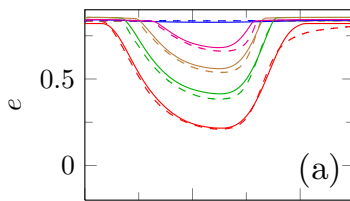

(a)

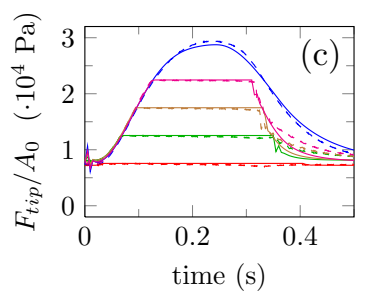

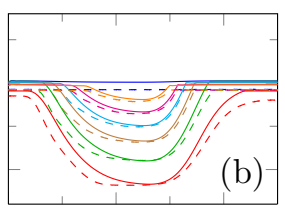

(b)

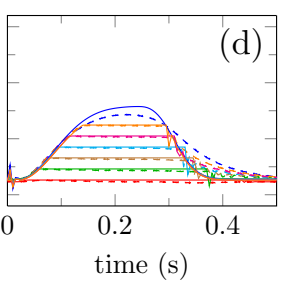

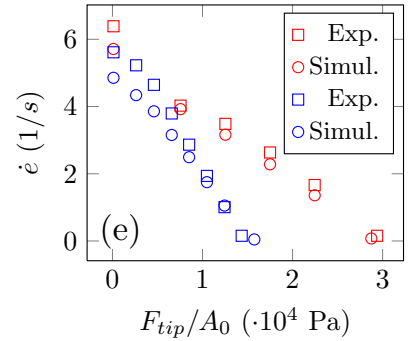

$F_{\text {tip }} / A_{0}\left(\cdot 10^{4} \mathrm{~Pa}\right)$

Fig. 3. Dynamic response of papillary muscle twitch contraction for high $(\mathrm{a}, \mathrm{c})$ and low $(\mathrm{b}, \mathrm{d})$ preloads: strain $(\mathrm{a}, \mathrm{b})$ and tension $(\mathrm{c}, \mathrm{d})$. Dashed lines: experimental data - Solid lines: simulations. (e): Peak velocity vs. force curve (with positive sign for shortening) corresponding to high (red) and low (blue) preloads. Parameters are: $R_{0}=1.3 \cdot 10^{-3} \mathrm{~m}, A_{0}=\pi R_{0}^{2}, L_{0}=5.10^{-3} \mathrm{~m}, \sigma_{0}=1.34 .10^{4} \mathrm{~Pa}$, $E s=5.10^{6} \mathrm{~Pa}, \alpha_{r}=0.12 \mathrm{~s}, C_{0}, C_{2}=9.10^{4}, C_{1}, C_{3}=4.10^{-3}, \eta, \mu=100$ Pa.s.
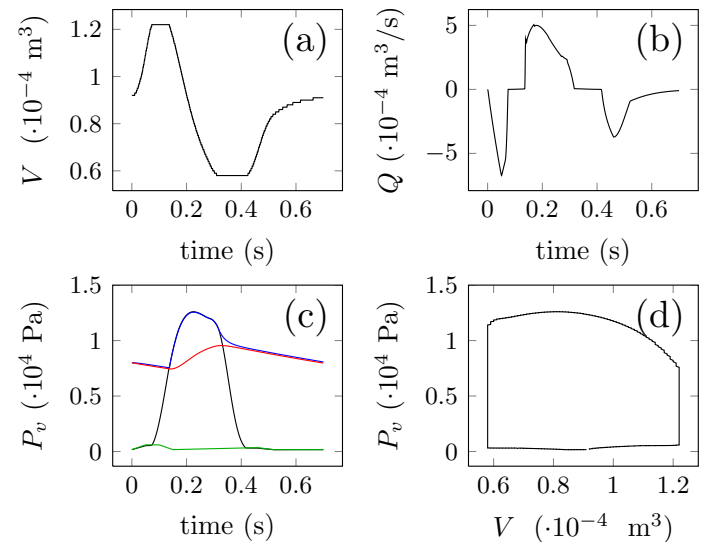

Fig. 4. Cardiac cycle obtained with the 0D-model. (a): Left ventricular volume. (b): Inflow (negative) and outflow (positive). (c): Ventricular (black), aortic (blue), distal (red) and atrial (green) pressures. The atrial pressure is a given function of time. (d): P-V diagram. Parameters are the same as in Fig.3 except $\sigma_{0}=1.1 .10^{5} \mathrm{~Pa}, R_{0}=0.0249 \mathrm{~m}$ and $d_{0}=5.10^{-3} \mathrm{~m}$. Additional parameters: $C_{p}=25.10^{-10}, R_{p}=10^{7}, C_{d}=0.18 .10^{-7}, R_{d}=8.25 .10^{7}, P_{v s}=10^{3} \mathrm{~Pa}$.

\section{Discussion and concluding remarks}

We see that our $1 \mathrm{D}$-model is able to most adequately reproduce single fiber experiments, and in particular the preload dependence of the Hill force-velocity curve, thanks to the Frank-Starling effect induced by the function $n_{0}$. Although 
the relaxation load dependence is well reproduced at high loading (see Fig.3 (c)), the low preload curves (see (d)) relax earlier than the experimental curves, which will motivate further modeling refinements. We also find that the single fiber experiments provide a most useful tool to calibrate the 1D-model, the parameters of which can then be used in a beating heart model, as evidenced with the 0D-model that produces realistic contraction cycles.

We have thus derived two reduced models of a full 3D formulation by only assuming simplified kinematics. The 1D-model successfully reproduces experiments on isolated fibers for different preloads and afterloads, notwithstanding the fact that the load-dependent relaxation warrants further refinement. Based on the parameters obtained from the calibration of the $1 \mathrm{D}$-model, the $0 \mathrm{D}$-model representing the left ventricle produces a realistic contraction cycle. All computation times for the reduced models are of a few seconds on a standard computer, which makes these models useful tools to calibrate a full 3D beating heart model, and / or to assess novel modeling ingredients based on specific experimental data. We point out that the 0D-reduction strategy could be easily extended to account for the behavior of 4 coupled cardiac cavities, when the corresponding calibration

data are available. Finally, these models allow simulations in nearly real time, which can be a great asset in the context of clinical applications.

\section{References}

1. O. Cazorla, J.-Y. Le Guennec, and E. White. Length-tension relationships of sub-epicardial and sub-endocardial single ventricular myocytes from rat and ferret hearts. J. Mol. Cell. Cardiol., 32(5):735-744, 2000.

2. D. Chapelle and K. J. Bathe. The Finite Element Analysis of Shells - Fundamentals. Springer, second edition, 2011.

3. D. Chapelle, P. Le Tallec, P. Moireau, and M. Sorine. An energy-preserving muscle tissue model: formulation and compatible discretizations. Int. J. Multiscale Com., 10(2):189-211, 2012.

4. G. A. Holzapfel and R. W. Ogden. Constitutive modelling of passive myocardium: a structurally based framework for material characterization. Philos. T. Roy. Soc. A, 367(1902):3445-3475, 2009.

5. P. J. Hunter, A. J. Pullan, and B. H. Smaill. Modeling total heart function. Annu. Rev. Biomed. Eng., 5:147-177, 2003.

6. A. F. Huxley. Muscle Structure and Theories of Contraction. Prog. Biophys. Mol. Bio., 7:258-318, 1957.

7. Y. C. Lecarpentier, L. H. Chuck, P. R. Housmans, N. M. De Clerck, and D. L. Brutsaert. Nature of load dependence of relaxation in cardiac muscle. Am J PhysiolHeart C, 1979.

8. J. Sainte-Marie, D. Chapelle, R. Cimrman, and M. Sorine. Modeling and estimation of the cardiac electromechanical activity. Comput. Struct., 84(28):1743-1759, 2006.

9. N. Smith et al. euHeart: personalized and integrated cardiac care using patientspecific cardiovascular modelling. Interface Focus, 1(3):349-364, 2011. 